\title{
CHARACTERIZATION OF EFFECTIVE MASSES AND OPTICAL GAIN IN STRAINED QUANTUM-WELL LASERS*
}

\author{
M.S. WARTAK, T.-M. MA \\ Department of Physics and Computing, Wilfrid Laurier University \\ Waterloo, Ontario, Canada N2L 3C5, \\ Z.-M. LI \\ Institute for Microstructural Sciences, National Research Council \\ Ottawa, Ontario, Canada K1A 0R6 \\ AND T. MAKINO \\ Advanced Technology Laboratory, Bell-Northern Research \\ Ottawa, Ontario, Canada K1Y $4 \mathrm{H7}$
}

\begin{abstract}
Valence-band structures, hole effective masses and optical gain have been calculated for strained-layer quantum-well laser structures. Results show a logarithmic relation between the peak optical gain and carrier concentration for all possible material compositions of the quaternary system.
\end{abstract}

PACS numbers: $73.20 . \mathrm{Dx}, 71.25 . \mathrm{Jd}$

Introduction of strain into quantum-well lasers produces many desirable effects such as lower threshold current and improved differential gain. However, the effect of strain and its modelling for $\mathrm{In}_{1-x} \mathrm{Ga}_{x} \mathrm{As}$ and $\mathrm{In}_{1-x} \mathrm{Ga}_{x} \mathrm{As}_{y} \mathrm{P}_{1-y}$ multiple quantum-well can be rather complicated. The complication of the model is worsened by the large parameter space of the above compounds. For a specific device application, there is infinite number of possible combinations for material compositions when strain is present. From the engineering point of view, simple and explicit formulas for the strain effect are desirable.

With this motivation in mind, we have used an efficient analytical model based on Luttinger-Kohn Hamiltonian to determine valence band structure, hole effective masses and optical gain for strained-layer quantum-well structures. Despite several recent efforts [1-3] the complete picture of hole effective masses is

*This work is supported by NSERC, BNR and WLU Office of Research. 
far from clear. The limited experimental results presented in [2] indicate a strong dependence of the in-plane effective masses of the heavy-hole and light-hole valence bands in quantum wells on their coupling. In general, the band mixing effect posts a much larger challenge in determining hole effective masses than electron effective masses in the conduction band. Negative effective masses at zone center can be predicted in some cases involving band mixing.

Our formulation is the same as the one used by Lee and Vassell [4]. Total Hamiltonian is expressed as $H=H_{\mathrm{LK}}+H_{\epsilon}$, where $H_{\mathrm{LK}}$ is the Luttinger-Kohn Hamiltonian and $H_{\epsilon}$ represents the strain Hamiltonian,

$$
\begin{aligned}
- & H_{\mathrm{LK}}=\frac{\hbar^{2}}{m_{0}}\left\{\frac{1}{2} \gamma_{1} K^{2}-\gamma_{2}\left[\left(J_{x}^{2}-\frac{1}{3} J^{2}\right) k_{x}^{2}+\text { c.p. }\right]\right. \\
& \left.-2 \gamma_{3}\left(\left\{J_{x}, J_{y}\right\} k_{x} k_{y}+\text { c.p. }\right)\right\}+V_{h}(z), \\
H_{\epsilon}= & D_{d}\left(\epsilon_{x x}+\epsilon_{y y}+\epsilon_{z z}\right)+\frac{2}{3} D_{u}\left[\left(J_{x}^{2}-\frac{1}{3} J^{2}\right) \epsilon_{x x}+\text { c.p. }\right] \\
& +\frac{4}{3} D_{u}^{\prime}\left(\left\{J_{x}, J_{y}\right\} \epsilon_{x y}+\text { c.p. }\right),
\end{aligned}
$$

where $m_{0}$ is the free-electron mass, $D_{d}, D_{u}$ and $D_{u}^{\prime}$ are deformation energies for valence bands, $\epsilon_{i j}$ are components of the strain tensor, $J_{i}$ are the angular momentum matrices, and $\{a, b\}=\frac{1}{2}(a b+b a)$.

In spin $J=\frac{3}{2}$ basis, the above Hamiltonian can be written as $4 \times 4$ matrix and block diagonalized into two equivalent $2 \times 2$ Hamiltonians. Standard boundary conditions are used as discussed by Burt [5]. The band edge effective mass was defined as inverse of second derivative with respect to wave vector. Optical gain was calculated using standard expressions [6] and Landsberg's gain broadening model has been used [7].

Figure 1 shows the results of heavy-hole effective masses in a strained quantum well as a function of $\mathrm{Ga}$ mole fraction for different well width for $\mathrm{In}_{1-x} \mathrm{Ga}_{x} \mathrm{As}$. Clearly, effective masses are decreasing as strain increases. For each curve a singular point exists where the calculated effective masses become infinity. This singularity corresponds to the point where the heavy-hole subband ceases to be the lowest subband and the band curvature at zone center changes from positive to negative.

In Fig. 2 we show the relation between the peak optical gain for $\mathrm{In}_{1-x} \mathrm{Ga}_{x} \mathrm{As}_{y} \mathrm{P}_{1-y}$ as a function of surface carrier concentration $N_{\mathrm{s}}\left(\right.$ in $\mathrm{m}^{-2}$ ) for compressive strain. Those points were fit by the following empirical formulas for peak optical gain $G$ (in $1 / \mathrm{m}$ ) as a function of compositional parameters $x$ and $y$ :

$$
G=1000 \times C_{1}(x, y) \ln N_{\mathrm{s}}+100 \times C_{0}(x, y)
$$

with

$$
\begin{aligned}
& C_{0}(x, y)=10 \times \sum_{j k} C_{0 j k} x^{j} y^{k}, \\
& C_{1}(x, y)=10 \times \sum_{j k} C_{1 j k} x^{j} y^{k}
\end{aligned}
$$

for compressive strain. Coefficients $C_{0 j k}$ and $C_{1 j k}$ are listed in Appendix. 


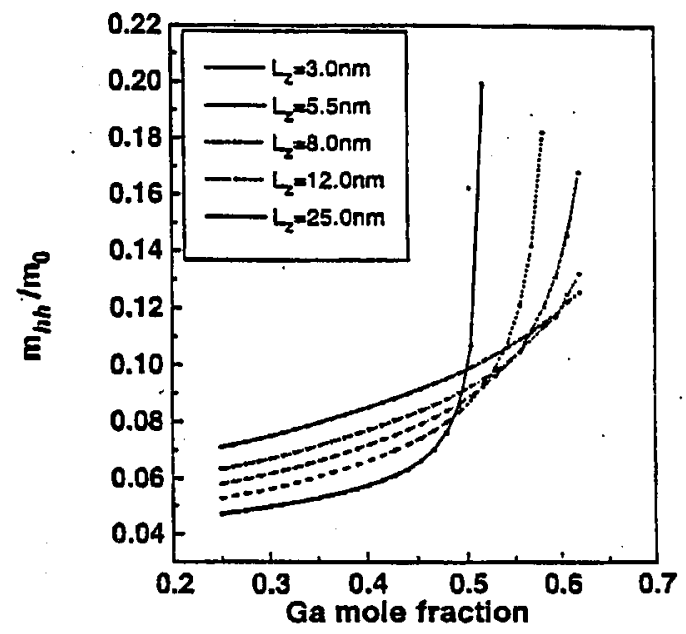

Fig. 1. Light-hole effective masses in strained quantum well as a function of Ga mole fraction and well width for tensile strain in $\mathrm{In}_{1-x} \mathrm{Ga}_{x}$ As material system.

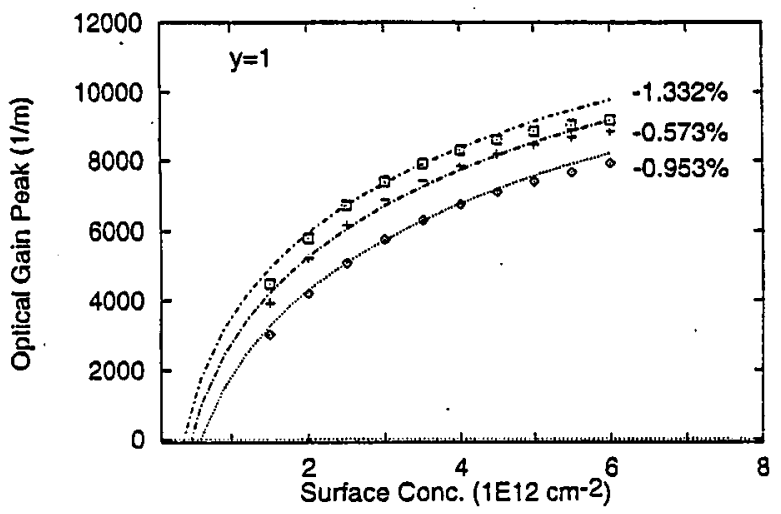

Fig. 2. Relations between optical gain and compressive strain for $\operatorname{In}_{1-x} \mathrm{Ga}_{x} \mathrm{As}_{y} \mathrm{P}_{1-y}$ single quantum-well lasers. The dots are from numerical calculation. The lines are from our empirical formulas.

One can show [8], based on work by Vahala and Zah [9], that the empirical relation between the peak optical gain and concentration can be derived analytically. The analytical formulas may be used directly in modeling and design of strained quantum-well lasers emitting at $1.55 \mu \mathrm{m}$. 


\section{Appendix}

Coefficients for empirical optical gain formulas.

$C_{0 j k}$ and $C_{1 j k}$ for compressive strain:

i) $C_{0 j k}$ :

$\begin{array}{cccc} & x^{0} & x^{1} & x^{2} \\ y^{0} & 0.135120 \times 10^{1} & -0.135267 \times 10^{2} & 0.281142 \times 10^{3} \\ y^{1} & -0.499414 \times 10^{1} & 0.525546 \times 10^{2} & -0.956443 \times 10^{3} \\ y^{2} & 0.577287 \times 10^{1} & -0.670164 \times 10^{2} & 0.108563 \times 10^{4} \\ y^{3} & -0.218948 \times 10^{1} & 0.276100 \times 10^{2} & -0.409816 \times 10^{3}\end{array}$

ii) $C_{1 j k}$ :

$$
\begin{array}{cccc} 
& x^{0} & x^{1} & x^{2} \\
y^{0} & -0.374309 \times 10^{1} & 0.337222 \times 10^{2} & -0.753185 \times 10^{3} \\
y^{1} & 0.137839 \times 10^{2} & -0.132791 \times 10^{3} & 0.256102 \times 10^{4} \\
y^{2} & -0.158640 \times 10^{2} & 0.170967 \times 10^{3} & -0.290614 \times 10^{4} \\
y^{3} & 0.599616 \times 10^{1} & -0.708517 \times 10^{2} & 0.109682 \times 10^{4}
\end{array}
$$

\section{References}

[1] B.K. Ridley, J. Appl. Phys. 68, 4667 (1990).

[2] L. Vina, L. Munoz, N. Mestres, E.S. Koteles, A. Ghiti, E.P. O'Reilly, D.C. Bertolet, K.M. Lau, Phys. Rev. B 47, 13926 (1993).

[3] T. Ishikawa, J.E. Bowers, IEEE J. Quantum Electron. 30, 562 (1994).

[4] Johnson Lee, M.O. Vassell, Phys. Rev. B 37, 8855 (1988).

[5] M.G. Burt, J. Phys. Condens. Matter 4, 6651 (1992).

[6] Z.-M. Li, M. Dion, S.P. McAlister, R.L. Williams, G.C. Aers, IEEE J. Quantum Electron. 29, 346 (1993).

[7] P.T. Landsberg, Phys. Status Solidi 15, 623 (1966).

[8] T. Makino, IEEE J. Quantum Electron., to be published.

[9] K.J. Vahala, C.E. Zah, Appl. Phys. Lett. 52, 1945 (1988). 